\title{
Effect of Forward Masking on Frequency Following Response as a Function of Age
}

\author{
Silvana Maria Sobral Griz ${ }^{1}$ Denise Costa Menezes ${ }^{1}$ Leonardo Gleygson Angelo Venâncio ${ }^{1}$ \\ Nathália Holanda da Fonsêca ${ }^{1}$ Thaís Ohanny do Nascimento ${ }^{1}$ Anne Karoline Lima de Araújo ${ }^{1}$ \\ Kelly Cristina Lira de Andrade ${ }^{2}$ Pedro de Lemos Menezes ${ }^{2}$
}

${ }^{1}$ Federal University of Pernambuco, Recife, Brazil

2 State University of Health Sciences of Alagoas, Maceió, Brazil

J Am Acad Audiol 2020;31:317-323.
Address for correspondence Silvana Maria Sobral Griz, Federal University of Pernambuco, Recife, Pernambuco, CEP 50740-020, Brazil (e-mail: silvana.griz@hotmail.com).
- aging

- forward masking

- frequency following response

- speech-evoked auditory brainstem response
Background Forward masking occurs when noise is presented before the target signal, making the latter difficult to be perceived. It is related to temporal auditory processing and consequently to speech recognition in noisy environments, which may decline with age. Interest in forward masking has grown in the last years. Studies investigate psychoacoustic and electrophysiological recordings in different age- groups.

Purpose The purpose of the study was to investigate the effect of forward masking on frequency following response (FFR) as a function of age.

Research Design Cross-sectional analytical observational study.

Study Sample We assessed 69 normal-hearing participants of both genders assigned to three groups: 40 young individuals (aged $18-25$ years, mean age $=22$ years 8 months), 21 middle-age individuals (aged 25-55 years, mean age $=37$ years 2 months), and 8 seniors (aged $<55$ years, mean age $=65$ years 3 months).

Intervention FFRs were recorded using the / da/ syllable with and without noise.

Data Collection and Analysis The / da/ syllable and speech-shaped noise were monaurally presented to the participants' right ears through ER-3a insert earphones. Electrodes were placed in M1 and M2 (-), Fz (+), and Fpz (ground). Acquisition occurred under two conditions: (1) the/da/ syllable presented without the noise and (2) the /da/ syllable presented 4 msec after the noise.

Results Data show that (1) considering the mean values of all participants, there was a significant latency delay of all waves (PV, A, PW, PX, PY, PZ, and O) when the /da/ syllable was presented $4 \mathrm{msec}$ after the masking noise as compared with the condition without noise, that is, forward masking occurred in all components of the FFR responses, and (2) for the youngest group and the middle-age group, forward masking was seen for all waves, except PX in the latter one; for the senior group, an irregular pattern was observed (presence of forward masking in PA, PY, $\mathrm{PZ}$, and $\mathrm{O}$ ). This pattern may be due to an aging effect on FFR responses even without noise presence, which makes it more difficult to identify forward masking effect in this population. Although it is well documented in the literature that forward masking increases with age, this is less evident on FFR recordings in the senior population.

Conclusions An aging effect was identified in FFR responses. Forward masking was identified in FFR responses of all groups but less evident in senior population.
Copyright $\odot 2020$ by the American Academy of Audiology. All rights reserved. Thieme Medical Publishers, Inc., 333 Seventh Avenue, New York, NY 10001, USA. Tel: +1(212) 760-0888.
DOI https://doi.org/ $10.3766 / j a a a .18104$ ISSN 1050-0545. 


\section{Introduction}

Listeners often face sound competition situations. Masking caused by a noisy environment compromises speech comprehension because noise can potentially degrade the temporal structures of acoustic information, making discourse unintelligible (Mehraei et al; ${ }^{17}$ Pienkowski) ${ }^{21}$. Recognizing speech in noisy settings is related to the ability of temporal auditory processing (Anderson and Kraus; ${ }^{1}$ Mamo et al: ${ }^{16}$ Fogerty et al) ${ }^{5}$, which is defined as the ability of the auditory system to perceive and differentiate stimuli and their acoustic characteristics over time (Terto and Lemos.) ${ }^{29}$ Results of psychophysical (Grose and Mamo) ${ }^{8}$ and electrophysiological tests (Grose and Mamo; ${ }^{7}$ Anderson and Kraus; ${ }^{1}$ Clinard and Tremblay) ${ }^{2}$ revealed deficits in temporal auditory processing as a function of age, manifesting as a decreased capacity to follow temporal changes in speech characteristics.

Several studies have investigated speech comprehension in a noisy environment, primarily in senior individuals, who, even with normal hearing, complain of misunderstanding speech in the presence of noise (Grose et al; ${ }^{10}$ Anderson and Kraus; ${ }^{1}$ Grose et al; ${ }^{11}$ Mamo et al; ${ }^{16}$ Schoof and Rosen; ${ }^{26}$ Fogerty et al $;{ }^{5}$ Hodge et al) ${ }^{14}$ Helfer and Vargo ${ }^{13}$ suggested that hearing skills might already be affected in middle-age individuals. However, there is no consensus regarding electrophysiological responses patterns for this population. Furthermore, studies of electrophysiological responses in the presence of noise for this population are also needed.

Temporal masking refers to changes in the perception of one sound caused by the presence of another, with enough duration and intensity to reduce the sensitivity of the target stimulus (Samelli and Schochat). ${ }^{24}$ It can occur simultaneously or not (backward masking and forward masking) (Necciari et al). ${ }^{18}$ To identify forward masking, noise must be presented "before" the target sound, causing a masking effect that persists for a few milliseconds (up to approximately $120 \mathrm{msec}$ ) after the presented noise has been ceased or attenuated, thereby changing perception of the target sound (Samelli and Schochat; ${ }^{24}$ DiGiovanni et al). ${ }^{4}$

Forward masking is related to temporal auditory processing and, consequently, speech perception in noisy environments. A common complaint of senior listeners, even those whose hearing thresholds are within normal values, is the difficulty to understand in noisy environments (Grose et al; ${ }^{9}$ Mamo et al; ${ }^{16}$ Schoof and Rosen; ${ }^{26}$ Fogerty et al; ${ }^{5}$ Pienkowski).21 Psychoacoustic (Grose and Mamo; ${ }^{8}$ Grose et al) ${ }^{9}$ and electrophysiological tests (Grose and Mamo; $;^{7}$ Anderson and Kraus; ${ }^{1}$ Clinard and Tremblay $)^{2}$ have been applied to understand speech in noise. Among the electrophysiological tests, auditory brainstem response (ABR) has been widely used, as it is an objective noninvasive procedure that assesses auditory pathway integrity at the brainstem level (Patel et al). ${ }^{20}$

Several stimuli can be used in the ABR procedure (Skoe and Kraus). ${ }^{27}$ The click stimulus produces more robust responses. Because of its transient characteristics and large spectrum, it activates a large number of neurons. With advances in auditory neuroscience, more complex diagnostic and assessment procedures were developed, including the ABR testing with verbal stimuli, such as a syllable. This type of stimuli may provide data on how speech is encoded. This test has been called frequency following response (FFR) (Sanfins and Colella-Santos). ${ }^{25}$ Among other speech stimuli, the syllable / $\mathrm{da}$ / has been mainly used because it is common to several languages, represents speech dynamicity, and reflects clear and replicable responses (Skoe and Kraus). ${ }^{27}$

Auditory-evoked responses for the syllable /da/ can be divided into the transient component, which corresponds to the consonant, and the sustained component, corresponding to the vowel (Grinstead). ${ }^{6}$ The transient component is a response of the onset of the syllable, characterized by the aperiodic modulation of the consonant, whereas sustained responses correspond to the harmonic and periodic structure of the vowel. Both components consist of complex waves that reflect neural activity, and their latencies and amplitudes can be measured and analyzed (Skoe and Kraus). ${ }^{27}$

A number of studies (Grose et al; ${ }^{10}$ Mamo et al; ${ }^{16}$ Schoof and Rosen; ${ }^{26}$ Fogerty et $a l ;{ }^{5}$ Hodge et al $)^{14}$ has assessed electrophysiological responses in old listeners in the presence of noise and exhibited age-related degradation in the temporal aspects of speech decoding. Regarding forward masking, for example, Hodge et al (2018) found it more evident for the senior population. However, evidence and behavior of forward masking in FFR responses for different age- groups remain unclear. Further comprehension of this effect will provide information on temporal processing aspects. Therefore, the aim of the present study was to analyze the effect of age and forward masking on FFR responses.

\section{Method}

This cross-sectional analytical observational study was conducted at the Audiology Laboratory, Speech and Hearing Science, Federal University of Pernambuco, between March 2016 and July 2017 and approved by the Institutional Ethics Committee on Human Research, under protocol number 1.727.677. The participants were recruited by convenience and advised of the study aims and procedures. The tests were scheduled according to the participants' and researchers' convenience. All participants singed a consent form.

Sixty-nine individuals of both gender, aged between 18 and 73 years (mean age $=34$ years), participated in the study. Forty-six (67\%) were female. Participants were divided into three groups: (a) 40 young individuals (aged 18-25 years, mean age $=22$ years 8 months), 21 middle-aged adults (aged $25-55$ years, mean age $=37$ years 2 months), and 8 seniors (aged $>55$ years, mean age $=65$ years 3 months).

The inclusion criteria were as follows: individuals without hearing complaints, with free external ear canal, and pure-tone audiometry thresholds \#25 dB HL, at frequencies between 250 and $8000 \mathrm{~Hz}$, except individuals who were aged $>60$ years, whose normal frequency ranged between 250 and $4000 \mathrm{~Hz}$, with "type A" tympa- nogram and ipsi- and contralateral acoustic reflexes present. Individuals with a history of ontological, neurological, or psychiatric diseases and cognitive problems, reported at the initial interview, were excluded. 


\section{Data Collection Methods}

Right-ear FFR was recorded using a synthetic syllable (/da/) and a speech-shaped noise as stimuli. The speech syllable had a duration of $40 \mathrm{msec}$, and it was

developed at the Northwestern University laboratory (Evanston, IL). It consists of a transient component, corresponding to the consonant $/ \mathrm{d} /$, and a sustained component, corresponding to the vowel /a/. The 100-msec masking noise was composed of Brazilian-Portuguese speech frequencies, and it was manufactured at the Laboratory of Hearing Sciences of the University of North Carolina at Chapel Hill. It had onset/offset ramps of $10 \mathrm{msec}$. Experiments were performed while participants sat on a reclined chair inside a treated sound booth. After the skin was cleaned with an abrasive paste, the negative electrodes were placed on the mastoid region (M1 and $\mathrm{M} 2$ ), the positive electrode at the Fz position, and the ground electrode at Fpz. ER-3A insert earphones were used to present the $/ \mathrm{da} /$ stimulus and the noise. FFR testing was conducted under two conditions: (a) unmasked condition, /da/ stimulus with no noise (NM-no masking) and (b) forward masking for 4-msec (FM) condition, /da/ stimulus presented $4 \mathrm{msec}$ after the masking noise. The stimuli rate was 3.77 stimuli/sec. The /da/ syllable was presented at 75-dB SPL and the speech-shaped noise at $80-\mathrm{dB}$ SPL. Intensity levels were thought considering the following reasons: the level of the / $\mathrm{da} /$ syllable had to be high enough to elicit a reliable response, but not too high, or it would not be susceptible to forward masking. The masker level had to be high enough to cause forward masking, but not too high, or it would be uncomfortably loud for the participant. Recording was finished after two replicated traces were performed with 2,000 stimuli each. Traces were summed, totalizing 4,000 averaged stimuli for each testing condition. The recording window was adjusted to $70 \mathrm{msec}$ and filters were between 50 and $1500 \mathrm{~Hz}$, with a gain of 100,000. According to the protocol of our audiology laboratory where the tests were applied ( - Figure 1), negative and positive peaks were identified on the final tracings of waves PV, A, PW, PX, PY, $\mathrm{PZ}$, and $\mathrm{O}$. Wave identification considered the principles used by Hodge et al (2018), who adopted the letter "P" to indicate wave positivity.

\section{Statistical Method}

The data were processed by Statistical Package for the Social Sciences (SPSS) software, version 21.0 (IBM Corp, Armonk, $\mathrm{NY}$ ), whose main measurements were FFR latencies. Analysis
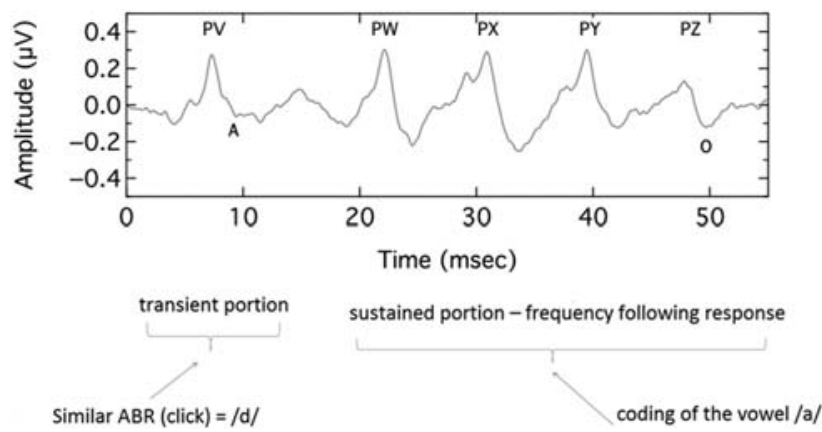

Fig. 1 FFR model of wave analysis. of variance for multiple measures was conducted. The Greenhouse-Geisser test was used to compare wave latencies (PV, A, PW, PX, PY, PZ, and O) within groups (between both conditions). The Sidak test was applied to compare both condition results among age-groups (young, middle age, and senior). Values were considered significant for $p<0.05$.

\section{Results}

-Figure 2 shows the wave latency of both conditions (unmasked and 4-msec forward masking) by age-group.

-Table 1 shows the means, standard deviations, and interval of normality (IN), in absolute latency values, by age-group (young, middle age, and seniors).

Difference between latency values of both conditions was calculated and called by df (B - A). Wave latencies, means, standard deviation values, and IN for both conditions, regardless of age, show a significant difference between the two conditions for all the measurements of the entire sample $(p<0.05)$. Specifically, for the young group, all the wave latencies were statistically different between the two test

A

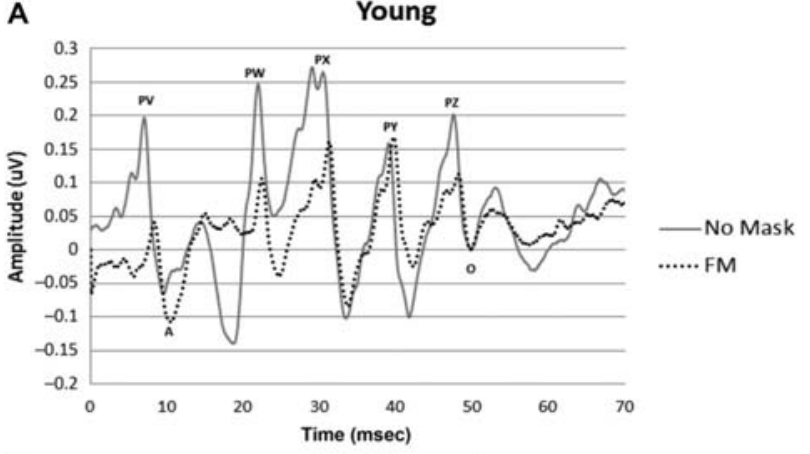

B Middle-aged

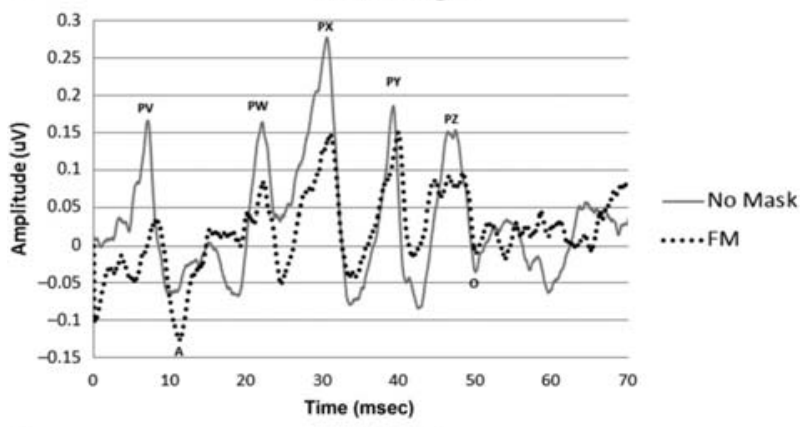
C Elderly

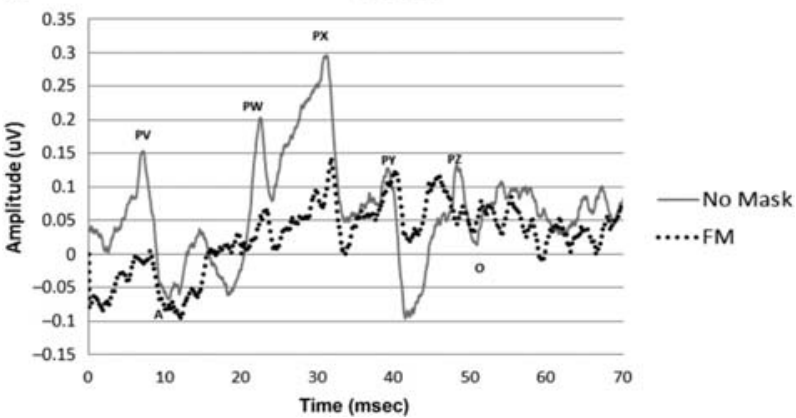

Fig. 2 Wave latencies for the unmasked and 4-msec forward masked conditions as function of age. 
Table 1 Wave Latency for the Unmasked and 4-msec Forward Masked Conditions by Age-Group

\begin{tabular}{|c|c|c|c|c|c|c|c|c|c|c|}
\hline \multirow[t]{2}{*}{ Wave } & \multicolumn{4}{|c|}{ Unmasked Condition (A) } & \multicolumn{4}{|c|}{ Forward Masking 4 msec (B) } & \multirow[t]{2}{*}{ df $B-A$} & \multirow[t]{2}{*}{$P$ Value* } \\
\hline & Mean & $1 \mathrm{DP}$ & $2.5 \mathrm{DP}$ & IN & Mean & $1 \mathrm{DP}$ & $2.5 \mathrm{DP}$ & IN & & \\
\hline \multicolumn{11}{|l|}{ Young } \\
\hline PV & 7.20 & 0.43 & 1.07 & $6.13-8.27$ & 8.18 & 0.78 & 1.95 & $6.23-10.13$ & 0.98 & 0.000 \\
\hline$A$ & 8.82 & 0.75 & 1.87 & 6.95-10.69 & 9.69 & 0.98 & 2.45 & $7.24-12.14$ & 0.87 & 0.000 \\
\hline PW & 22.0 & 1.10 & 2.75 & $19.25-24.75$ & 22.6 & 1.65 & 4.12 & $18.48-26.72$ & 0.6 & 0.033 \\
\hline $\mathrm{PX}$ & 30.3 & 1.74 & 4.35 & $25.95-34.65$ & 31.2 & 1.62 & 4.05 & $27.15-35.25$ & 0.9 & 0.004 \\
\hline PY & 39.0 & 1.68 & 4.20 & $34.8-43.2$ & 40.2 & 2.49 & 6.22 & $33.98-46.42$ & 1.2 & 0.001 \\
\hline $\mathrm{PZ}$ & 47.8 & 2.55 & 6.37 & $41.43-54.17$ & 48.8 & 3.16 & 7.9 & $40.9-56.7$ & 1 & 0.008 \\
\hline $\mathrm{O}$ & 49.2 & 3.02 & 7.55 & $41.65-56.75$ & 50.0 & 3.29 & 8.22 & $41.78-58.22$ & 0.8 & 0.041 \\
\hline \multicolumn{11}{|c|}{ Middle age } \\
\hline PV & 7.24 & 0.43 & 1.07 & $6.17-8.31$ & 8.75 & 0.78 & 1.95 & $6.8-10.7$ & 1.51 & 0.000 \\
\hline A & 8.59 & 0.75 & 1.87 & $6.72-10.46$ & 10.23 & 0.97 & 2.42 & $7.81-12.65$ & 1.64 & 0.000 \\
\hline PW & 22.0 & 0.67 & 1.67 & $20.33-23.67$ & 22.8 & 1.01 & 2.52 & $20.28-25.32$ & 0.8 & 0.001 \\
\hline PX & 30.8 & 1.06 & 2.65 & $28.15-33.45$ & 31.2 & 0.99 & 2.47 & $28.73-33.67$ & 0.4 & 0.145 \\
\hline PY & 39.4 & 1.03 & 2.57 & $36.83-41.97$ & 40.2 & 1.52 & 3.8 & $36.4-44$ & 0.8 & 0.007 \\
\hline $\mathrm{PZ}$ & 47.7 & 1.56 & 3.9 & $43.8-51.6$ & 49.1 & 1.94 & 4.85 & $44.25-53.95$ & 1.4 & 0.000 \\
\hline $\mathrm{O}$ & 49.1 & 1.85 & 4.62 & $44.48-53.72$ & 50.3 & 2.01 & 5.02 & $45.28-55.32$ & 1.2 & 0.000 \\
\hline \multicolumn{11}{|c|}{ Seniors } \\
\hline PV & 7.64 & 0.42 & 1.05 & $6.59-8.69$ & 8.00 & 0.78 & 1.95 & $6.05-9.95$ & 0.36 & 0.188 \\
\hline A & 9.38 & 0.75 & 1.87 & $7.51-11.25$ & 10.50 & 0.97 & 2.42 & $8.08-12.92$ & 1.12 & 0.004 \\
\hline PW & 22.8 & 0.67 & 1.67 & $21.13-24.47$ & 23.1 & 1.01 & 2.52 & $20.58-25.62$ & 0.3 & 0.518 \\
\hline$P X$ & 31.5 & 1.06 & 2.65 & $28.85-34.15$ & 31.9 & 0.99 & 2.47 & $29.43-34.37$ & 0.4 & 0.381 \\
\hline PY & 40.5 & 1.02 & 2.55 & $37.95-43.05$ & 42.3 & 1.52 & 3.8 & $38.5-46.1$ & 1.8 & 0.001 \\
\hline $\mathrm{PZ}$ & 49.7 & 1.56 & 3.9 & $45.8-53.6$ & 52.1 & 1.94 & 4.85 & $47.25-56.95$ & 2.4 & 0.000 \\
\hline $\mathrm{O}$ & 51.6 & 1.85 & 4.62 & $46.98-56.22$ & 54.9 & 2.01 & 5.02 & $49.88-59.92$ & 3.3 & 0.000 \\
\hline
\end{tabular}

*SIDAK test.

conditions $(p<0.05)$; for the middleage population, only the wave PX showed no significant difference between both conditions $(p=0.145)$; for the senior group, the waves PV $(p=0.188)$, PW $(p=0.518)$, and PX (0.381) showed no difference between both conditions.

- Table 2 shows the difference between wave latencies in both testing conditions, considering combinations (A-B) among age-groups.

For the unmasked condition, there were significant differences in latencies between (a) the young and seniors for PV $(p=0.033), \mathrm{PW}(p=0.027), \mathrm{PX}(p=0.041), \mathrm{PY}(p=0.006), \mathrm{PZ}$ $(p=0.026)$, and $O$ waves $(p=0.015)$ (except for wave A) and (b) the middleage group and seniors for PW $(p=0.015)$, PY $(p=0.036), \mathrm{PZ}(p=0.016)$, and $\mathrm{O}$ waves $(p=0.007)$ (except for waves PV, A, and PX). There were no significant differences between latencies of the young and middleage groups.

For stimulus presentation $4 \mathrm{msec}$ after the masking noise, there were significant differences between (a) the young and seniors for PY $(p=0.012), \mathrm{PZ}(p=0.001)$, and $\mathrm{O}$ waves $(p=0.000)$; (b) the middle-age group and seniors for PY $(p=0.007), \mathrm{PZ}(p=0.002)$, and $\mathrm{O}$ waves $(p=0.000)$; and (c) the young and middle-age group only for the PV wave $(p=0.026)$.

\section{Discussion}

Speech comprehension difficulties in the presence of noise and associated with forward masking have been reported in normal-hearing individuals (Grose et al). ${ }^{16}$ Forward masking has been investigated by using psychoacoustic testing (Grose and Mamo $;{ }^{8}$ Grose et al; ${ }^{11}$ Mehraei et al; ${ }^{17}$ Niemczak and Vander ${ }^{19}$ and auditory-evoked potentials (Grose and Mamo; ${ }^{7}$ Clinard and Tremblay; ${ }^{2}$ Mamo et al; ${ }^{16}$ Hodge et al ${ }^{14}$ in different age populations. The influence of noise on speech perception is an important research field, as it is known that in social noisy environments, forward masking may render speech sound information inaudible or poorly perceived. This scenario is worse for senior listeners (Hodge et al, 2018). ${ }^{14}$

Fogerty et al (2017) have demonstrated an aging effect in psychoacoustic responses for consonants and vowels in conditions of simultaneous and nonsimultaneous masking in young adults and seniors. Their young participants 
Frequency Following Response as a Function of Age Griz et al. 321

Table 2 Differences between Wave Latencies in Both Testing Conditions According to Combinations among Age-Groups

\begin{tabular}{|c|c|c|c|c|c|}
\hline \multirow[b]{2}{*}{ PV } & \multirow{2}{*}{$\begin{array}{l}\text { Age-Group (I') } \\
\text { NM }\end{array}$} & \multirow{2}{*}{$\begin{array}{l}\text { Age-Group (I") } \\
\text { Young }\end{array}$} & \multirow{2}{*}{$\frac{\text { l'-l"' }}{\text { Middle age }}$} & \multicolumn{2}{|c|}{ Significance* } \\
\hline & & & & -0.040 & 0.981 \\
\hline & & & Senior & $-0.436^{\dagger}$ & $0.033^{*}$ \\
\hline & & Middle age & Senior & -0.396 & 0.089 \\
\hline & FM & Young & Middle age & $-0.572^{\dagger}$ & $0.026^{*}$ \\
\hline & & & Senior & 0.173 & 0.922 \\
\hline & & Middle age & Senior & 0.744 & 0.075 \\
\hline \multirow[t]{6}{*}{ A } & NM & Young & Middle age & 0.224 & 0.615 \\
\hline & & & Senior & -0.564 & 0.161 \\
\hline & & Middle age & Senior & -0.788 & 0.051 \\
\hline & FM & Young & Middle age & -0.539 & 0.128 \\
\hline & & & Senior & -0.812 & 0.103 \\
\hline & & Middle age & Senior & -0.273 & 0.878 \\
\hline \multirow[t]{6}{*}{ PW } & NM & Young & Middle age & 0.019 & 1.000 \\
\hline & & & Senior & $-0.811^{\dagger}$ & $0.027^{*}$ \\
\hline & & Middle age & Senior & $-0.830^{\dagger}$ & $0.015^{*}$ \\
\hline & FM & Young & Middle age & -0.224 & 0.886 \\
\hline & & & Senior & -0.473 & 0.645 \\
\hline & & Middle age & Senior & -0.248 & 0.914 \\
\hline \multirow[t]{6}{*}{ PX } & NM & Young & Middle age & -0.467 & 0.494 \\
\hline & & & Senior & $-1.204^{\dagger}$ & $0.041^{*}$ \\
\hline & & Middle age & Senior & -0.737 & 0.281 \\
\hline & FM & Young & Middle age & 0.066 & 0.996 \\
\hline & & & Senior & -0.659 & 0.359 \\
\hline & & Middle age & Senior & -0.725 & 0.239 \\
\hline \multirow[t]{7}{*}{ PY } & NM & Young & Middle age & -0.369 & 0.650 \\
\hline & & & Senior & $-1.491^{\dagger}$ & $0.006^{*}$ \\
\hline & & Middle age & Young & 0.369 & 0.650 \\
\hline & & & Senior & $-1.122^{\dagger}$ & $0.036^{*}$ \\
\hline & FM & Young & Middle age & 0.016 & 1.000 \\
\hline & & & Senior & $-2.041^{\dagger}$ & $0.012^{*}$ \\
\hline & & Middle age & Senior & $-2.058^{\dagger}$ & $0.007^{*}$ \\
\hline \multirow[t]{6}{*}{$\mathrm{PZ}$} & NM & Young & Middle age & 0.032 & 1.000 \\
\hline & & & Senior & $-1.884^{\dagger}$ & 0.026 \\
\hline & & Middle age & Senior & $-1.916^{\dagger}$ & 0.016 \\
\hline & FM & Young & Middle age & -0.329 & 0.945 \\
\hline & & & Senior & $-3.307^{\dagger}$ & $0.001^{*}$ \\
\hline & & Middle age & Senior & $-2.978^{\dagger}$ & $0.002^{*}$ \\
\hline \multirow[t]{6}{*}{$\mathrm{O}$} & NM & Young & Middle age & 0.108 & 0.997 \\
\hline & & & Senior & $-2.399^{\dagger}$ & $0.015^{*}$ \\
\hline & & Middle age & Senior & $-2.507^{\dagger}$ & $0.007^{*}$ \\
\hline & FM & Young & Middle age & -0.344 & 0.944 \\
\hline & & & Senior & $-4.918^{\dagger}$ & $0.000^{*}$ \\
\hline & & Middle age & Senior & $-4.574^{\dagger}$ & 0.000 \\
\hline
\end{tabular}

*Adjust for multiple comparisons: Sidak test.

†Statistically significant difference. 
showed higher speech recognition scores than the elderly in the nonsimultaneous masking condition. Seniors needed a longer time interval between speech and noise to obtain speech recognition scores equivalent to young people.

Considering auditory-evoked potentials, latency shifts in noise presentation characterizes forward masking and may be due to the delay of the auditory nervous system to recover from a previous masking stimulus. Studies on forward masking (Grose et al $;{ }^{11}$ Fogerty et al $;{ }^{5}$ Mehraei et al $;{ }^{17}$ Hodge et al ${ }^{14}$ have reported the influence of the noise on evoked responses, regardless of the stimulus, population, and acquisition criteria that have been used. In the present data, forward masking was identified on all waves (PV, A, PX, PY, PW, PZ, and O waves) for all groups.

Similar results were found by Russo et al (2004), who have recorded FFR in two conditions: stimulus alone and stimulus with simultaneous noise. They found bigger latency shifts at the transient portion of the response than at the sustained portion. These findings suggest that the sustained component of the syllable $(/ \mathrm{a} /)$ is less vulnerable to the masking effect because it remained temporarily stable and easily identifiable despite the presence of noise. Other studies (Johnson et al; ${ }^{15}$ Song et al; ${ }^{28}$ Fogerty et al $)^{5}$ reinforce the idea that the transient portion of the response is more vulnerable to the effect of the noise. However, Schoof and Rosen (2016) reported changes not only at the transient but also at the sustained portion, when the syllable /da/ was used. In the present data, higher latency shifts were also found on the transient portion of the response.

The transient component of the response may be more affected by masking because it is closer (in time) to the noise, and therefore, the fibers of the auditory system have less time to recover from the effect of the noise. In addition to that, it is the part of the response corresponding to the consonant of the stimulus (d), which is briefer and less intense than the sustained component of the stimulus, the vowel (a).

Hodge et al (2018) showed a relation between forward masking and temporal processing deficits associated with age. They have recorded FFR in several conditions that varied in time lag between the presented noise and the syllable. Their data show an increase in forward masking as the time lag between the presentation of the syllable /da/ and masking noise decreased, especially for senior listeners. This aging effect in forward masking findings was not seen in the present study. Our data do not show more evidence of forward masking in seniors possibly because FFR responses of the syllable alone show less synchrony patterns of responses. However, an aging effect is shown in FFR responses without the presence of noise, as latencies of young and middle-aged participants were different from those of the senior group. In other words, an aging effect has caused different patterns of FFR responses among the groups without the presence of noise, and this may have cloaked forward masking identification in the seniors group.

A greater effect of forward masking in senior listeners is consistent with a slower recovery from the noise effect. Agerelated decreases for processing vowels in noise may occur because of poorer coding of vowel temporal periodicity. Walton et al (1999) investigated the recovery time of brainstem auditory responses for click and tone burst stimuli in young and senior normal-hearing individuals. They observed that the longer the time interval between stimulus and noise, the greater the likelihood of latency returning to the unmasked values. Both young participants and seniors exhibited a pattern of latency recovery, but the recovering was slower in the senior people.

Mamo et al (2016) reported that the spectral and harmonic components of the auditory-evoked responses of the seniors are reduced in relation to the stimulus because of the decline in neural synchrony. Breaks in the temporal wave of the stimulus occur in the encoding of periodic and complex signals, which may result in speech comprehension problems, especially in noisy environments.

In behavioral tests that involve cortical skills, forward masking was observed in seniors (Coffey et al; ${ }^{3}$ Schoof and Rosen; ${ }^{26}$ Niemczak and Vander). ${ }^{19}$ In a psychoacoustic test with modulated masking, Grose et al (2016) observed that seniors show lower forward masking magnitude than young and middleaged individuals. Then, changes in temporal processing related with age can be seen in different situations of testing.

Temporal processing deficits may start early in the elderly. In the present data, although there was no statistical difference in unmasked responses between the young and middleage groups, the latter, when compared with seniors, exhibited a difference only in PW, PY, and PZ waves. When looking into latencies of the forward masking condition, the middleage group showed significant delay in PV, as compared with the young group, although most of the wave latencies have not significantly differed from those found in the young group, and despite not being significantly similar to the seniors. This age-related qualitative analysis may indicate a decline in temporal auditory processing even before senescence. Other studies (Helfer and Vargo; ${ }^{13}$ Ruggles et al; ${ }^{22}$ Grose et al; ${ }^{9}$ Helfer $)^{12}$ report that when masking occurs at longer intervals in relation to the stimulus, middle-aged listeners perform worse than their younger counterparts.

\section{Conclusions}

Forward masking in FFR responses was identified in the young, middle-age, and senior groups, but it was less evident in the senescent auditory system corresponding to the senior population. This may be due to this group's nature of the responses, which already exhibited an inconsistent pattern without masking.

An aging effect was observed in FFR recordings without the presence of a masking noise. These findings suggest that the onset of a decline in temporal processing can already be seen in middle-aged adults.

\section{Abbreviations}

ABR auditory brainstem response

FFR frequency following response

IN interval of normality

Notes

This study was financed in part by the Coordination for the Improvement of Higher Education Personnel, Brazil (CAPES), Finance Code 001. 


\section{References}

1 Anderson S, Kraus N. The potential role of the cABR in assessment and management of hearing impairment. Int J Otolaryngol 2013 2013:1-10

2 Clinard CG, Tremblay KL. Aging degrades the neural encoding of simple and complex sounds in the human brainstem. J Am Acad Audiol 2013;24(07):590-599

3 Coffey EB, Herholz SC, Chepesiuk AM, Baillet S, Zatorre RJ. Cortical contributions to the auditory frequency-following response revealed by MEG. Nat Commun 2016;7:11070

4 DiGiovanni JJ, Lynch EE, Nagaraj NK, Ries DT. Dominance of persistence over adaptation in forward masking. Atten Percept Psychophys 2018;80(07):1863-1869

5 Fogerty D, Bologna WJ, Ahlstrom JB, Dubno JR. Simultaneous and forward masking of vowels and stop consonants: effects of age, hearing loss, and spectral shaping. J Acoust Soc Am 2017;141(02): 1133-1143

6 Grinstead L. Frequency Following Response (FFR): Examining the Variability of this Complex response. PhD Thesis. Towson UniversityTowson, MD2017

7 Grose JH, Mamo SK. Processing of temporal fine structure as a function of age. Ear Hear 2010;31(06):755

8 Grose JH, Mamo SK. Frequency modulation detection as a measure of temporal processing: age-related monaural and binaural effects. Hear Res 2012;294(1-2):49-54

9 Grose JH, Mamo SK, Buss E, Hall JW. Temporal processing deficits in middle age. Am J Audiol 2015;24(02):91-93

10 Grose JH, Mamo SK, Hall JW III. Age effects in temporal envelope processing: speech unmasking and auditory steady state responses. Ear Hear 2009;30(05):568

11 Grose JH, Menezes DC, Porter HL, Griz S. Masking period patterns \& forward masking for speech-shaped noise: age-related effects. Ear Hear 2016;37(01):48

12 Helfer KS. Competing speech perception in middle age. Am J Audiol 2015;24(02):80-83

13 Helfer KS, Vargo M. Speech recognition and temporal processing in middle-aged women. J Am Acad Audiol 2009;20(04):264-271

14 Hodge SE, Menezes DC, Brown KD, Grose JH. Forward masking of the speech-evoked auditory brainstem response. Otol Neurotol 2018;39(02):150-157

15 Johnson KL, Nicol TG, Kraus N. Brain stem response to speech: a biological marker of auditory processing. Ear Hear 2005;26(05): 424-434
16 Mamo SK, Grose JH, Buss E. Speech-evoked ABR: effects of age and simulated neural temporal jitter. Hear Res 2016;333:201-209

17 Mehraei G, Gallardo AP, Shinn-Cunningham BG, Dau T. Auditory brainstem response latency in forward masking, a marker of sensory deficits in listeners with normal hearing thresholds. Hear Res 2017;346:34-44

18 Necciari T, Laback B, Savel S, Ystad S Balazs P, Meunier S, Kronland-Martinet R. Auditory time-frequency masking for spectrally and temporally maximally-compact stimuli. PLoS One 2016;11 (11):e0166937

19 Niemczak CE, Vander Werff KR. Informational masking effects on neural encoding of stimulus onset and acoustic change. Ear Hear 2018;40(01):156-167

20 Patel K, Shah C, Mehta H, Patel H, Dixit G, Thakor N. Study of interpeak latencies of waveforms of brainstem auditory evoked potentials in normal healthy persons. Natl J Physiol Pharm Pharmacol 2017;7(08):831

21 Pienkowski M. On the etiology of listening difficulties in noise despite clinically normal audiograms. Ear Hear 2017;38(02):135

22 Ruggles D, Bharadwaj H, Shinn-Cunningham BG. Why middleaged listeners have trouble hearing in everyday settings. Curr Biol 2012;22(15):1417-1422

23 Russo N, Nicol T, Musacchia G, Kraus N. Brainstem responses to speech syllables. Clin Neurophysiol 2004;115(09):2021-2030

24 Samelli AG, Schochat E. Auditory processing, temporal resolution and gap detection test: literature review. Rev CEFAC 2008;10(03): 369-377

25 Sanfins MD, Colella-Santos MF. Frequency following response. In: Menezes PL, Andrade KCL, Frizzo ACF, Carnauba ATL, Lins OG, eds. Tratado de Eletrofisiologia para a Audiologia. Ribeirão Preto, SP: Book Toy; 2019:97-116

26 Schoof T, Rosen S. The role of age-related declines in subcortical auditory processing in speech perception in noise. J Assoc Res Otolaryngol 2016;17(05):441-460

27 Skoe E, Kraus N. Auditory brainstem response to complex sounds: a tutorial. Ear Hear 2010;31(03):302

28 Song JH, Skoe E, Banai K, Kraus N. Perception of speech in noise: neural correlates. J Cogn Neurosci 2011;23(09):2268-2279

29 Terto SDSM, Lemos SMA. Temporal aspects of auditory: knowledge production in four national journals. Rev CEFAC 2011;13(05):926-936

30 Walton J, Orlando M, Burkard R. Auditory brainstem response forward-masking recovery functions in senior humans with normal hearing. Hear Res 1999;127(1-2):86-94 